\title{
DIRECT-TO-HOME TELEVISION SERVICES IN EUROPE
}

\author{
Branimir Jakšić ${ }^{1}$, \\ Mile Petrović ${ }^{1}$, \\ Petar Spalević ${ }^{1}$, \\ Bojana Milosavljević2 \\ Marko Smilić ${ }^{3}$ \\ ${ }^{1}$ University of Pristina, \\ Faculty of Technical Sciences, \\ Kosovska Mitrovica, Serbia \\ ${ }^{2}$ Singidunum University, \\ Faculty of Informatics and Computing, \\ Belgrade, Serbia \\ ${ }^{3}$ University of Pristina, \\ Faculty of Natural Sciences and Mathematics, \\ Kosovska Mitrovica, Serbia
}

Correspondence:

Branimir Jakšić

e-mail:

branimir.jaksic@pr.ac.rs

\begin{abstract}
:
The paper describes the main characteristics and the principle of distribution of signals Direct-to-Home service. The most important characteristics of DTH providers in Europe: satellite, satellite positions, the number of multiplexes, the DVB standard, compression, standard and coding system are presented. Also, the change in the number of SDTV and HDTV DTH providers for five years since 2000 is given. The characteristics that provide DTH services in former Yugoslavia are presented, as well as the structure of the TV channels that are distributed as part of the DTH services in the states of former Yugoslavia.
\end{abstract}

Key words:

Direct-to-Home (DTH), satelit, satellite positions, SDTV, HDTV.

\section{Acknowledgment}

This work was done within the research project of the Ministry of Science and Technological Development of Serbia III47016 and TR35026.

\section{INTRODUCTION}

Direct-to-Home (DTH) technology refers to the process of broadcasting the satellite TV signal and other services designed exclusively for home reception with personal antennas. This technology was previously signed as a Direct Broadcast Satellite (DBS) technology (Pattan, 1993; Valenti, 2011; Chaplin, 1992). DTH technology has been developed to compete with tv services cable operators, broadcasting high-quality satellite signals with a large number of TV channels. The use of satellites has significant advantages for broadcasting and distribution of television programs, which are primarily reflected in the service area of a large territory, the construction of unnecessary infrastructure and the ability to transfer more channels with the same satellite (Valenti, 2011). On the other hand, due to a much greater technical capacity than other forms of transmission, satellite transmission is ideal for development of HDTV.

Thanks to the rapid development of digital technology, DTH operators all over the world, beside the permanent increase of number of TV channels in SD (Sdandard Definition) and HD (High Definition) format, introduce new services along with the interactive applications, video on demand, satellite internet and others. DTH services are broadcasted from high power satellites so that the reception is available directly into homes by using smaller antenna diameter of 60 to $90 \mathrm{~cm}$. The most suitable 
and the most present transfer of DTH services is in $\mathrm{Ku}$ band, although there are services in $\mathrm{C}$ and Ka band. TV channels that are broadcasted via DTH in digital format are generally encrypted so that the receiver/decoder of protected channels is required to access them (Tirro, 1993). DTH transmission excludes local cable operators completely, because the user is directly connected with the service provider.

\section{TRANSMISSION OF SIGNALS WITH DTH TECHNOLOGY}

There are five major components in DTH systems: the signal source, the emission center (uplink antenna), satellite, downlink antenna and receiver (Figure 1).

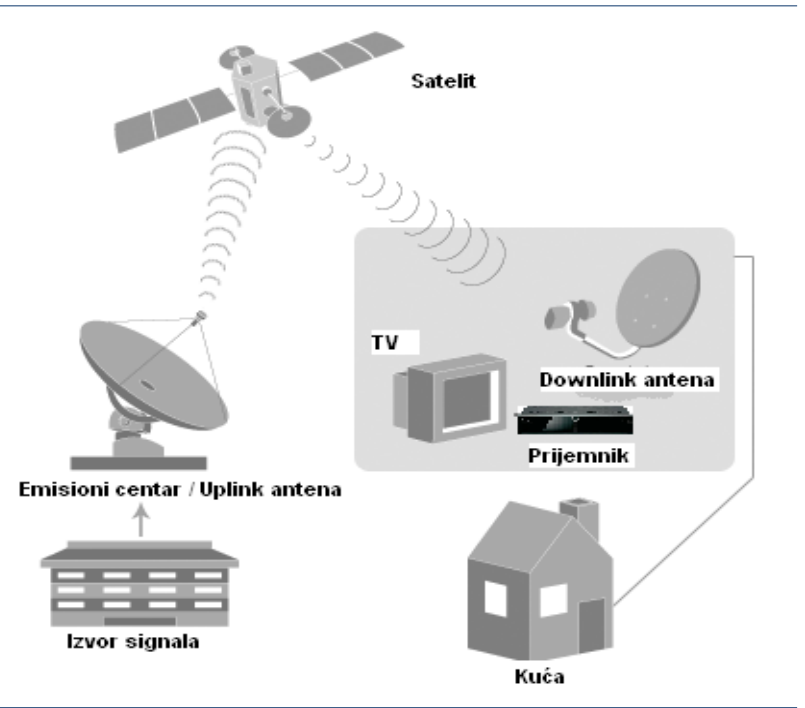

Figure 1. DTH system.

Signals are sent from the ground station to a satellite uplink antenna via the emission of the object whose dimensions are a diameter of 9 to 12 meters. On one satellite exists a large number of transponders whose task is to receive high-frequency modulated signals that are broadcasted fromterrestrial broadcasting station, and also to amplify them at another frequency and broadcast them towards the Earth (Petrović, 2007). Table 1 shows the frequency bands for uplink and downlink (Valenti, 2011).

\begin{tabular}{ccc}
\hline $\begin{array}{c}\text { Frequency } \\
\text { band }\end{array}$ & Downlink & Uplink \\
\hline $\mathrm{C}$ & $3.7-4.2 \mathrm{GHz}$ & $5.925-6.425 \mathrm{GHz}$ \\
\hline $\mathrm{Ku}$ & $11.7-12.2 \mathrm{GHz}$ & $14.0-14.5 \mathrm{GHz}$ \\
\hline $\mathrm{Ka}$ & $17.7-21.2 \mathrm{GHz}$ & $27.5-31.0 \mathrm{GHz}$ \\
\hline
\end{tabular}

Table 1. Frequency band for uplink i downlink
Downlink signals, attenuated after propagation through long sections, are collected with downlink parabolic satellite antenna.Dimensions of receiving antennas depend on the strength of the satellite transponder and satellite coverage area. In DTH services, receiving antennas have a diameter of 60 to $90 \mathrm{~cm}$. Receiving antenna reflects signals into a single point that low-noise block converter - LNB enhances (about 50dB, 100,000 times) and converts it from $\mathrm{C}$ and $\mathrm{Ku}$ band to the frequency range of the receiver.This converted and amplified signal is led by using coaxial cable, which should have little attenuation in the satellite receiver for further processing (Petrović, 2007; Mirabito and Morgenstern, 2004).

The first satellite television transmission was in the analogous format. The main characteristic of analogous distribution is that one TV channel was using a single frequency, or a satellite transponder. This method of distribution resulted in the high cost of leasing transponders, and thus a smaller number of broadcast TV channels in relation to digital transmission. Because of the stated characteristics, DTH services in analogous technics were very poor and with very small number of TV channels. Unlike analogous reception, higher quality digital signal transmission enables that from one transponder is broadcasted a larger number of programs that form a multiplex.The result had a direct impact on the development of DTH services all over the world, especially as the price of a digital broadcasting signal significantly diminished and therefore the number of television and radio channels, as well as other services, increased significantly.

The development of digital telecommunications enabled, besides the Standard Definition Television (SDTV), also the use of high-definition television - HDTV. HDTV is a technology that offers picture and sound quality significantly higher than the traditional technology of visual and sound display (analogous PAL, NTSC, SECAM, and digital SDTV) (Pechard et al., 2006). In recent years, in addition to HDTV, the first Ultra High Definition Television has appeared (UHDTV) which included $4 \mathrm{~K}$ UHDTV and 8K UHDTV with two times larger, or four times larger number of pixels in regard to Full HDTV (Marcotte, 2012).

The transfer of television video signal in its uncompressed formats requires a very large throughput flow that is outside the flow supported by modern technology. Therefore, different algorithms are used to compress video signals. The two most popular compression standards for digital TV signals are MPEG-2 and MPEG-4 AVC (H.264) (Haykin). Lately, higher quality MPEG-4 
standard is more and more in use compared to the previous MPEG-2 standard. For UHDTV, HEVC compression standardwas adopted by the ITU (International Telecommunication Union).

DVB-S (Digital Video Broadcasting - Satellite) is the oldest DVB standard, which was adopted in 1994 by the European Telecommunications Standards Institute (ETSI). It is a satellite transmission of digitized audio and video content by a system of geostationary satellites and adequate receivers. The second generation of this standard - DVB-S2 has a larger capacity, it uses more efficient modulation and MPEG-4 / H.264 compression. DVB-S2 delivers 30\% better performance compared to DVB-S, which in combination with the MPEG-4 / H.264 compression provides the ability that HDTV program broadcasts with the same flow that was formerly required for SDTV (Mignone et al., 2011).

\section{DTH PLATFORM DEVELOPMENT IN EUROPE}

Satellite television services in Europe started to use the Ku band in late 1980s. In 1988, Luxembourg company SES Astra has launched the first satellite medium power Astra 1A, which was designed for reception of signals by individual antennas of a smaller diameter $(90 \mathrm{~cm})$. Before DTH service in digital format, in the mid-1990s, the signals from the satellites were broadcasted analogously. In analogous format, the most famous DTH platform was British Sky Analogue (1990-2001) which broadcasted over 30 encrypted TV channels from Astra satellites at $19.2^{\circ}$ East. In addition to Sky Analogue, two significant analogous DTH platforms in Europe were the Scandinavian Canal + and Viasat. The other analogous DTH services were comprised of one or several encrypted TV channels for France, Germany, Spain, the Netherlands, Turkey and Russia.

The first digital DTH platform in Europe began distributing TV channels in the second half of the 1990s in France, Germany, Netherlands, Italy, Spain and the UK. Table 2 provides the main characteristics of the DTH platform which are broadcasted in Europe (KingOfSat, 2015; LingSat, 2015). Table 2 shows that all countries in Europe have DTH services. In some countries there are several DTH providers (Romania, Hungary, France, Bulgaria, Serbia, Croatia ...). In previous years, there has been a joint of several DTH platforms into one, as is the case with Sky Italia, which was formed in 2003. The joint of the Italian DTH platform Tele + and Stream TV,
Movistar +2003 was created by joining the Spanish DTH platform via Digital and CanalSatelite Digital, NC + was created by joining N Poland i Cyfra+.

Some DTH providers offer services from multiple satellites, such as Canal Digitaal, TV Vlaandern, Orange France and Bis TV. The most popular coding systems are Nagravision, Viaccess, Conax and VideoGuard. DTH providers such as Canal Digtiaal, Vlaandern TV, Canal Sat France, NC +, Bis TV, Sky Link, UPC Direct and KabelKiosk use two or three coding systems. DTH platforms to the greatest extent use DVB-S2 stanndard and MPEG-4 compression for broadcasting TV channels. Some operators continue to use the DVB-S standard and MPEG-2 compression with a tendency of switching to DVB-S2 / MPEG-4. For HDTV are exclusively used DVB-S2 / MPEG-4. The largest number of DTH platforms is broadcasted from the two most popular satellite positions in Europe $\left(13^{\circ}\right.$ East and $19.2^{\circ}$ East). A large number of providers that are located on the same satellite positions perform mutual "exchange" of TV by including a channel codingin a number of systems. This leads to saving in the capacity of transponders or multiplexes.

Table 2 presents the number of multiplexes and satellite transponders, which are owned by European DTH providers. DTH providers beside from their multiplex, also broadcast TV channels from multiplexes of other DTH providers with whom they exchange TV channels, or with a certain compensation. Orange Polska DTH platform with $13^{\circ}$ East does not have its own multiplex,but provides its services from the trasnponder of other DTH operators. The largest number of multiplexes has Sky UK (61), then Canal Digital (30) and Viasat (29).

Table 3 shows the number of SDTV and HDTV channels for DTH operators in Europe at the end of (31 of December) 2000, 2005, 2010 and 2015.

The number of TV channels increases from year to year, but since 2005 appear the first HDTV channels (Jakšić et al., 2014). At the end of 2015, the highest number of SDTV channels is offered by Sky Sports provider (495), followed by NTV Plus (227) Digi TV (217) Viasat (210), Sky Italia (196) ... The largest number of TV channels is offered by Canalsat France (144), Sky Sports (95) Sky Deutschland (85). Besides its own SDTV and HDTV channels, DTH providers in its offer include the so-called free (free-to-air) SDTV and HDTV channels that are distributed from the same satellite. Such is the case with satellite position $28.2^{\circ} \mathrm{E}, 19.2^{\circ} \mathrm{E}$ and $13^{\circ} \mathrm{E}$ from which broadcasts a large number of unencrypted SDTV and HDTV channels. 


\begin{tabular}{|c|c|c|c|c|c|c|c|}
\hline DTH provider & Country & Start & Pozition & Satellite & $\begin{array}{l}\text { No. } \\
\text { MUX }\end{array}$ & Standard / Compression & Type Coding \\
\hline Vivacom & Bulgaria & 2010 & $45.0^{\circ} \mathrm{E}$ & Intelsat 12 & 6 & DVB-S2, MPEG-4 & VideoGurad \\
\hline D-Smart & Turkey & 2007 & $42.0^{\circ} \mathrm{E}$ & Turksat $2 \mathrm{~A} / 3 \mathrm{~A} / 4 \mathrm{~A}$ & 8 & $\begin{array}{l}\text { DVB-S, MPEG-2 za SD } \\
\text { DVB-S2, MPEG-4 za HD }\end{array}$ & VideoGurad \\
\hline Bulsatcom & Bulgaria & 2004 & $39.0^{\circ} \mathrm{E}$ & Hellas Sat 2 & 5 & $\begin{array}{l}\text { DVB-S, MPEG-2 za SD } \\
\text { DVB-S2, MPEG-4 za HD }\end{array}$ & BulCrypt \\
\hline Polaris Media & Serbia & 2010 & $39.0^{\circ} \mathrm{E}$ & Hellas Sat 2 & 1 & $\begin{array}{l}\text { DVB-S, MPEG-2 } \\
\text { DVB-S2, MPEG-4 }\end{array}$ & BulCrypt \\
\hline Dolce TV & Romania & 2006 & $39.0^{\circ} \mathrm{E}$ & Hellas Sat 2 & 9 & $\begin{array}{l}\text { DVB-S, MPEG-2 za SD } \\
\text { DVB-S2, MPEG-4 za HD }\end{array}$ & VideoGurad \\
\hline NTV Plus & Russia & 1999 & $36.0^{\circ} \mathrm{E}$ & Eutelsat 36A/36B & 22 & $\begin{array}{l}\text { DVB-S, MPEG-2 } \\
\text { DVB-S2, MPEG-4 }\end{array}$ & Viaccess \\
\hline Tricolor TV & Russia & 2005 & $36.0^{\circ} \mathrm{E}$ & Eutelsat 36A/36B & 18 & $\begin{array}{l}\text { DVB-S, MPEG-2 } \\
\text { DVB-S2, MPEG-4 }\end{array}$ & DRE-Crypt \\
\hline MagtiSat & Georgia & 2012 & $31.5^{\circ} \mathrm{E}$ & Astra 5B & 4 & DVB-S2, MPEG-4 & BulCrypt \\
\hline Orange Romnaia & Romania & 2013 & $31.5^{\circ} \mathrm{E}$ & Astra 5B & 7 & DVB-S2, MPEG-4 & Viaccess \\
\hline Sky UK & UK & 1998 & $28.2^{\circ} \mathrm{E}$ & Astra $2 \mathrm{E} / 2 \mathrm{~F} / 2 \mathrm{G}$ & 61 & $\begin{array}{l}\text { DVB-S, MPEG-2 za SD } \\
\text { DVB-S2, MPEG-4 za HD }\end{array}$ & VideoGurad \\
\hline SkyLink & $\begin{array}{l}\text { Czech R., } \\
\text { Slovakia }\end{array}$ & 2007 & $23.5^{\circ} \mathrm{E}$ & Astra 3B & 6 & $\begin{array}{l}\text { DVB-S, MPEG-2 } \\
\text { DVB-S2, MPEG-4 }\end{array}$ & Cryptoworks, Irdeto \\
\hline $\begin{array}{l}\text { Canal Digitaal } \\
(23.5 \mathrm{E})\end{array}$ & Netherlands & 1996 & $23.5^{\circ} \mathrm{E}$ & Astra 3B & 7 & DVB-S2, MPEG-4 & $\begin{array}{l}\text { Mediaguard, Nagra- } \\
\text { vision, Viaccess }\end{array}$ \\
\hline $\begin{array}{l}\text { Canal Digitaal } \\
(19.2 \mathrm{E})\end{array}$ & Netherlands & 1996 & $19.2^{\circ} \mathrm{E}$ & $\begin{array}{c}\text { Astra } \\
1 \mathrm{KR} / 1 \mathrm{~L} / 1 \mathrm{M} / 1 \mathrm{~N}\end{array}$ & 2 & $\begin{array}{l}\text { DVB-S, MPEG-2 } \\
\text { DVB-S2, MPEG-4 }\end{array}$ & $\begin{array}{l}\text { Mediaguard, Nagra- } \\
\text { vision, Viaccess }\end{array}$ \\
\hline $\begin{array}{l}\text { TV Vlaanderen } \\
(23.5 \mathrm{E}) \\
\end{array}$ & Belgium & 2006 & $23.5^{\circ} \mathrm{E}$ & Astra 3B & 7 & DVB-S2, MPEG-4 & $\begin{array}{l}\text { Mediaguard, Nagra- } \\
\text { vision, Viaccess }\end{array}$ \\
\hline $\begin{array}{l}\text { TV Vlaanderen } \\
(19.2 \mathrm{E})\end{array}$ & Belgium & 2006 & $19.2^{\circ} \mathrm{E}$ & $\begin{array}{c}\text { Astra } \\
1 \mathrm{KR} / 1 \mathrm{~L} / 1 \mathrm{M} / 1 \mathrm{~N}\end{array}$ & 2 & $\begin{array}{l}\text { DVB-S, MPEG-2 } \\
\text { DVB-S2, MPEG-4 }\end{array}$ & $\begin{array}{l}\text { Mediaguard, Nagra- } \\
\text { vision, Viaccess }\end{array}$ \\
\hline Sky Deutschland & Germany & 1996 & $19.2^{\circ} \mathrm{E}$ & $\begin{array}{c}\text { Astra } \\
1 \mathrm{KR} / 1 \mathrm{~L} / 1 \mathrm{M} / 1 \mathrm{~N}\end{array}$ & 12 & $\begin{array}{l}\text { DVB-S, MPEG-2 } \\
\text { DVB-S2, MPEG-4 }\end{array}$ & VideoGurad \\
\hline Movistar+ Astra & Spain & 1997 & $19.2^{\circ} \mathrm{E}$ & $\begin{array}{c}\text { Astra } \\
1 \mathrm{KR} / 1 \mathrm{~L} / 1 \mathrm{M} / 1 \mathrm{~N}\end{array}$ & 17 & $\begin{array}{l}\text { DVB-S, MPEG-2 za SD } \\
\text { DVB-S2, MPEG-4 za HD }\end{array}$ & $\begin{array}{l}\text { Mediaguard, Nagra- } \\
\text { vision }\end{array}$ \\
\hline Canal Sat France & France & 1996 & $19.2^{\circ} \mathrm{E}$ & $\begin{array}{c}\text { Astra } \\
1 \mathrm{KR} / 1 \mathrm{~L} / 1 \mathrm{M} / 1 \mathrm{~N}\end{array}$ & 22 & $\begin{array}{l}\text { DVB-S, MPEG-2 za SD } \\
\text { DVB-S2, MPEG-4 za HD }\end{array}$ & $\begin{array}{l}\text { Mediaguard, Nagra- } \\
\text { vision, Viaccess }\end{array}$ \\
\hline $\begin{array}{l}\text { Orange France } \\
(19.2 \mathrm{E})\end{array}$ & France & 2008 & $19.2^{\circ} \mathrm{E}$ & $\begin{array}{c}\text { Astra } \\
1 \mathrm{KR} / 1 \mathrm{~L} / 1 \mathrm{M} / 1 \mathrm{~N}\end{array}$ & 1 & $\begin{array}{l}\text { DVB-S, MPEG-2 za SD } \\
\text { DVB-S2, MPEG-4 za HD }\end{array}$ & Viaccess \\
\hline AustriaSat & Austria & 2010 & $19.2^{\circ} \mathrm{E}$ & $\begin{array}{c}\text { Astra } \\
1 \mathrm{KR} / 1 \mathrm{~L} / 1 \mathrm{M} / 1 \mathrm{~N}\end{array}$ & 1 & $\begin{array}{l}\text { DVB-S, MPEG-2 } \\
\text { DVB-S2, MPEG-4 }\end{array}$ & Irdeto \\
\hline Total TV & $\begin{array}{l}\text { Serb., Croatia, } \\
\text { Slovenia, B\&H, } \\
\text { Maced., Mont. }\end{array}$ & 2006 & $16^{\circ} \mathrm{E}$ & Eutelsat 16A & 11 & $\begin{array}{l}\text { DVB-S, MPEG-2 za SD } \\
\text { DVB-S2, MPEG-4 za HD }\end{array}$ & VideoGurad \\
\hline Max TV & Croatia & 2010 & $16^{\circ} \mathrm{E}$ & Eutelsat 16A & 3 & DVB-S2, MPEG-4 & Nagravision \\
\hline Vip Sat & Croatia & 2013 & $16^{\circ} \mathrm{E}$ & Eutelsat 16A & 2 & DVB-S2, MPEG-4 & Conax \\
\hline DigitAlb & Albania & 2004 & $16^{\circ} \mathrm{E}$ & Eutelsat 16A & 4 & DVB-S2, MPEG-4 & Conax \\
\hline Tring Digital & Albania & 2008 & $16^{\circ} \mathrm{E}$ & Eutelsat 16A & 2 & DVB-S2, MPEG-4 & Conax \\
\hline Mtel & Bulgaria & 2014 & $16^{\circ} \mathrm{E}$ & Eutelsat 16A & 3 & DVB-S2, MPEG-4 & Conax \\
\hline
\end{tabular}




\begin{tabular}{|c|c|c|c|c|c|c|c|}
\hline Antik Sat & Slovakia & 2015 & $16^{\circ} \mathrm{E}$ & Eutelsat 16A & 3 & DVB-S2, MPEG-4 & Conax \\
\hline Sky Italia & Italy & 1997 & $13^{\circ} \mathrm{E}$ & $\begin{array}{c}\text { Hot Bird } \\
13 \mathrm{~B} / 13 \mathrm{C} / 13 \mathrm{D}\end{array}$ & 25 & $\begin{array}{l}\text { DVB-S, MPEG-2 za SD } \\
\text { DVB-S2, MPEG-4 za HD }\end{array}$ & VideoGurad \\
\hline Nova & Greece, Cyprus & 1999 & $13^{\circ} \mathrm{E}$ & $\begin{array}{c}\text { Hot Bird } \\
13 \mathrm{~B} / 13 \mathrm{C} / 13 \mathrm{D}\end{array}$ & 4 & $\begin{array}{l}\text { DVB-S, MPEG-2 } \\
\text { DVB-S2, MPEG-4 }\end{array}$ & Irdeto \\
\hline Cifrowy Polsat & Poland & 1999 & $13^{\circ} \mathrm{E}$ & $\begin{array}{c}\text { Hot Bird } \\
13 \mathrm{~B} / 13 \mathrm{C} / 13 \mathrm{D}\end{array}$ & 6 & DVB-S2, MPEG-4 & Nagravision \\
\hline $\mathrm{NC}+$ & Poland & 1998 & $13^{\circ} \mathrm{E}$ & $\begin{array}{c}\text { Hot Bird } \\
13 \mathrm{~B} / 13 \mathrm{C} / 13 \mathrm{D}\end{array}$ & 10 & $\begin{array}{l}\text { DVB-S, MPEG-2 } \\
\text { DVB-S2, MPEG-4 }\end{array}$ & $\begin{array}{l}\text { Conax, } \\
\text { Mediaguard }\end{array}$ \\
\hline Orange Polska & Poland & 2008 & $13^{\circ} \mathrm{E}$ & $\begin{array}{c}\text { Hot Bird } \\
13 \mathrm{~B} / 13 \mathrm{C} / 13 \mathrm{D}\end{array}$ & 0 & $\begin{array}{l}\text { DVB-S, MPEG-2 } \\
\text { DVB-S2, MPEG-4 }\end{array}$ & Viaccess \\
\hline $\begin{array}{l}\text { Orange France } \\
(13 E)\end{array}$ & France & 2008 & $13^{\circ} \mathrm{E}$ & $\begin{array}{c}\text { Hot Bird } \\
13 \mathrm{~B} / 13 \mathrm{C} / 13 \mathrm{D}\end{array}$ & 3 & DVB-S2, MPEG-4 & Viaccess \\
\hline Bis TV (13E) & France & 2007 & $13^{\circ} \mathrm{E}$ & $\begin{array}{c}\text { Hot Bird } \\
13 \mathrm{~B} / 13 \mathrm{C} / 13 \mathrm{D}\end{array}$ & 2 & DVB-S2, MPEG-4 & $\begin{array}{l}\text { Viaccess, Medi- } \\
\text { aguard }\end{array}$ \\
\hline KabelKiosk & Germany & 2009 & $9^{\circ} \mathrm{E}$ & Eutelsat 9A & 10 & DVB-S2, MPEG-2, MPEG-4 & Conax, VideoGuard \\
\hline AustriaSat Magy & y Hungary & 2008 & $9^{\circ} \mathrm{E}$ & Eutelsat 9A & 2 & DVB-S2, MPEG-4 & Conax, Irdeto \\
\hline OTE TV & Greece & 2012 & $9^{\circ} \mathrm{E}$ & Eutelsat 9A & 6 & DVB-S2, MPEG-4 & VideoGurad \\
\hline DigiTurk & Turkey & 2000 & $7^{\circ} \mathrm{E}$ & Eutelsat 7A & 21 & $\begin{array}{l}\text { DVB-S, MPEG-2 za SD } \\
\text { DVB-S2, MPEG-4 za HD }\end{array}$ & Cryptoworks, Irdeto \\
\hline Viasat & $\begin{array}{l}\text { Swed., Norw., } \\
\text { Finla., Denm., } \\
\text { Lithuania, Latvia, } \\
\text { Eston. }\end{array}$ & 1999 & $4.8^{\circ} \mathrm{E}$ & Astra 4A, SES 5 & 29 & $\begin{array}{l}\text { DVB-S, MPEG-2 za SD } \\
\text { DVB-S2, MPEG-4 za HD }\end{array}$ & VideoGurad \\
\hline Viasat Ukraine & Ukraine & 2008 & $4.8^{\circ} \mathrm{E}$ & Astra 4A, SES 5 & 2 & DVB-S2, MPEG-4 & VideoGurad \\
\hline Canal Digital & $\begin{array}{l}\text { Swed., Norw., } \\
\text { Finland, Denmark }\end{array}$ & 1998 & $0.8^{\circ} \mathrm{W}$ & $\begin{array}{c}\text { Thor } 5 / 6 \text {, Intelsat } \\
10-02\end{array}$ & 30 & $\begin{array}{l}\text { DVB-S, MPEG-2 } \\
\text { DVB-S2, MPEG-4 }\end{array}$ & Conax \\
\hline UPC Direkt & $\begin{array}{l}\text { Hungary, Czech } \\
\text { R., Slovakia }\end{array}$ & 2000 & $0.8^{\circ} \mathrm{W}$ & $\begin{array}{c}\text { Thor } 5 / 6 \text {, Intelsat } \\
10-02\end{array}$ & 9 & $\begin{array}{l}\text { DVB-S, MPEG- } 2 \text { za SD } \\
\text { DVB-S2, MPEG- } 4 \text { za HD }\end{array}$ & $\begin{array}{l}\text { Conax, Cryptow- } \\
\text { orks, Irdeto, Nagra- } \\
\text { vision }\end{array}$ \\
\hline Focus Sat & Romania & 2004 & $0.8^{\circ} \mathrm{W}$ & $\begin{array}{c}\text { Thor } 5 / 6 \text {, Intelsat } \\
10-02\end{array}$ & 5 & $\begin{array}{l}\text { DVB-S, MPEG-2 za SD } \\
\text { DVB-S2, MPEG-4 za HD }\end{array}$ & Conax \\
\hline Digi TV & $\begin{array}{l}\text { Roma., Hung., } \\
\text { Slovak, Czech R., } \\
\text { Serbia }\end{array}$ & 2004 & $0.8^{\circ} \mathrm{W}$ & $\begin{array}{c}\text { Thor } 5 / 6 \text {, Intelsat } \\
10-02\end{array}$ & 16 & $\begin{array}{l}\text { DVB-S, MPEG- } 2 \text { za SD } \\
\text { DVB-S2, MPEG- } 4 \text { za HD }\end{array}$ & Nagravision \\
\hline T-Home & Hungary & 2008 & $4^{\circ} \mathrm{W}$ & Amos $2 / 3$ & 6 & DVB-S2, MPEG-4 & Conax \\
\hline $\begin{array}{l}\text { Orange France } \\
(5 \mathrm{~W})\end{array}$ & France & 2008 & $5^{\circ} \mathrm{W}$ & Eutelsat 5 West A & 1 & $\begin{array}{l}\text { DVB-S, MPEG-2 za SD } \\
\text { DVB-S2, MPEG-4 za HD }\end{array}$ & Viaccess \\
\hline Bis TV $(5 \mathrm{~W})$ & France & 2007 & $5^{\circ} \mathrm{W}$ & Eutelsat 5 West A & 2 & $\begin{array}{l}\text { DVB-S, MPEG-2 za SD } \\
\text { DVB-S2, MPEG-4 za HD }\end{array}$ & Viaccess \\
\hline Fransat & France & 2009 & $5^{\circ} \mathrm{W}$ & Eutelsat 5 West A & 8 & $\begin{array}{l}\text { DVB-S, MPEG-2 za SD } \\
\text { DVB-S2, MPEG-4 za HD }\end{array}$ & Viaccess \\
\hline $\begin{array}{l}\text { Movistar+ } \\
\text { Hispasat }\end{array}$ & Spain & 1997 & $30^{\circ} \mathrm{W}$ & Hispasat 1D/1E & 10 & DVB-S, MPEG-2 & Nagravision \\
\hline NOS & Portugal & 2000 & $30^{\circ} \mathrm{W}$ & Hispasat $1 \mathrm{D} / 1 \mathrm{E}$ & 5 & $\begin{array}{l}\text { DVB-S, MPEG-2 } \\
\text { DVB-S2, MPEG-4 }\end{array}$ & Nagravision \\
\hline MEO & Portugal & 2008 & $30^{\circ} \mathrm{W}$ & Hispasat $1 \mathrm{D} / 1 \mathrm{E}$ & 4 & DVB-S2, MPEG-4 & $\begin{array}{l}\text { Mediaguard, Na- } \\
\text { gravis. }\end{array}$ \\
\hline
\end{tabular}

Table 2. Characteristic DTH platforms in Evrope 


\begin{tabular}{|c|c|c|c|c|c|c|c|c|}
\hline \multirow{2}{*}{ DTH provider } & \multicolumn{2}{|c|}{2000.} & \multicolumn{2}{|c|}{2005.} & \multicolumn{2}{|c|}{2010.} & \multicolumn{2}{|c|}{2015.} \\
\hline & SD & HD & $\mathrm{SD}$ & $\mathrm{HD}$ & SD & HD & SD & $\mathrm{HD}$ \\
\hline Vivacom & - & - & - & - & 80 & - & 97 & 14 \\
\hline D-Smart & - & - & - & - & 75 & 9 & 81 & 51 \\
\hline Bulsatcom & - & & 31 & - & 66 & 6 & 89 & 10 \\
\hline Polaris Media & - & - & - & - & 43 & 2 & 63 & 4 \\
\hline Dolce TV & - & - & - & - & 85 & 6 & 106 & 16 \\
\hline NTV Plus & 36 & - & 62 & - & 168 & 9 & 227 & 34 \\
\hline Tricolor TV & - & - & 12 & - & 77 & - & 186 & 39 \\
\hline MagtiSat & - & - & - & - & - & - & 106 & 2 \\
\hline Orange Romnaia & - & - & - & - & - & - & 76 & 40 \\
\hline Sky UK & 268 & - & 474 & - & 523 & 50 & 495 & 95 \\
\hline SkyLink & - & - & - & - & 60 & 12 & 92 & 41 \\
\hline Canal Digitaal 23.5E & - & - & - & - & 31 & 21 & 52 & 33 \\
\hline Canal Digitaal 19.2E & 21 & - & 36 & 1 & 47 & 1 & 24 & 2 \\
\hline TV Vlaanderen23.5E & - & - & - & - & - & - & 39 & 24 \\
\hline TV Vlaanderen19.2E & - & - & - & - & 47 & 2 & 23 & 5 \\
\hline Sky Deutschland & 46 & - & 50 & - & 74 & 13 & 75 & 85 \\
\hline Movistar+ Astra & 50 & - & 91 & - & 87 & 21 & 107 & 44 \\
\hline Canal Sat France & 77 & - & 124 & 1 & 171 & 21 & 68 & 144 \\
\hline Orange France (19.2E) & - & - & - & - & - & - & 57 & 19 \\
\hline AustriaSat & - & - & - & - & - & - & 14 & 15 \\
\hline Total TV & - & - & - & - & 100 & 3 & 123 & 35 \\
\hline Max TV & - & - & - & - & 50 & - & 73 & 9 \\
\hline Vip Sat & - & - & - & - & - & - & 65 & 13 \\
\hline DigitAlb & - & - & 19 & - & 56 & 6 & 55 & 24 \\
\hline Tring Digital & - & - & - & - & 24 & - & 45 & 7 \\
\hline Mtel & - & - & - & - & - & - & 57 & 27 \\
\hline Antik Sat & - & - & - & - & - & - & 37 & 10 \\
\hline Sky Italia & 64 & - & 152 & - & 204 & 40 & 196 & 69 \\
\hline Nova & 24 & - & 44 & - & 49 & 4 & 51 & 14 \\
\hline Cifrowy Polsat & 17 & - & 38 & - & 70 & 14 & 93 & 60 \\
\hline $\mathrm{NC}+$ & 19 & - & 57 & - & 101 & 14 & 98 & 78 \\
\hline Orange Polska & - & - & - & - & 61 & 7 & 67 & 52 \\
\hline Orange France (13E) & - & - & - & - & 49 & 2 & 69 & 8 \\
\hline Bis TV (13E) & - & - & - & - & 35 & - & 34 & 6 \\
\hline KabelKiosk & - & - & - & - & 91 & 11 & 54 & 35 \\
\hline AustriaSat Magyarország & - & - & - & - & 31 & 7 & 45 & 13 \\
\hline OTE TV & - & - & - & - & - & - & 66 & 31 \\
\hline DigiTurk & 61 & - & 83 & - & 118 & 14 & 131 & 48 \\
\hline Viasat & 24 & - & 92 & - & 196 & 17 & 210 & 48 \\
\hline Viasat Ukraine & - & - & - & - & 40 & - & 37 & 7 \\
\hline Canal Digital & 78 & - & 116 & 1 & 190 & 26 & 166 & 69 \\
\hline UPC Direkt & 36 & - & 58 & - & 101 & 11 & 137 & 24 \\
\hline Focus Sat & - & - & 34 & - & 62 & - & 74 & 7 \\
\hline Digi TV & - & - & 58 & - & 167 & - & 217 & 22 \\
\hline T-Home & - & - & - & - & 82 & 4 & 104 & 23 \\
\hline Orange France (5W) & - & - & - & - & 66 & 2 & 71 & 12 \\
\hline Bis TV $(5 \mathrm{~W})$ & - & - & - & - & 31 & - & 31 & 7 \\
\hline Fransat & - & - & - & - & 48 & 4 & 76 & 29 \\
\hline Movistar+ Hispasat & 78 & - & 86 & - & 90 & - & 115 & - \\
\hline NOS & 18 & - & 81 & - & 88 & 11 & 61 & 16 \\
\hline MEO & - & - & - & - & 88 & 8 & 91 & 15 \\
\hline
\end{tabular}

Table 3. SD and HD TV channels number. 


\section{DTH PLATFORM IN FORMER YUGOSLAVIA}

Thefirst DTH platform for the countries of the former Yugoslavia was a package of TV channels called SeeMe. The platform began with its work in January 2005, with a dozen TV channels. In the first half of 2006, DTH platform Total TV began its work, and initially it was intended only for Serbia, but later expanded the business to Montenegro, Bosnia and Herzegovina, Croatia, Slovenia and Macedonia. At the end of 2006, the Romanian DTH platform took users of Seemore package and launched its services in Serbia and Croatia (DigiTV, 2015). Since 2013, Digi TV provided services only to users in Serbia, and users from Croatia were assumed by newly formed Vip Sat DTH platform (VipSat, 2015). In 2010, two more DTH platforms started - Polaris Media in Serbia and Max TV in Croatia (KingOfSat, 2015).

Characteristics of DTH providers in the former Yugoslavia can be seen in Table 2. Even three DTH platforms: Total TV, Max TV and Vip Sat are distributed from the satellite position 16 degrees East, a Polaris Media from $39^{\circ}$ East and Digi TV from $1^{\circ}$ West. Platforms Vip Sat and Max TV exclusively use DVB-S2/MPEG-4 standard for the transmission of TV channels, while Total TV uses DVB-S2/MPEG-4 for TV, and for SDTV, it uses DVB-S/ MPEG-2 (Petrović et al., 2014).

Table 4 gives the number of SDTV and HDTV channels at the end of year for DTH platforms that provide services in countries of former Yugoslavia. For DTH platform Digi TV were taken into account only TV channels that were broadcasted in Serbia and Croatia, not counting the TV channels for other markets. The number of TV channels increased from year to year, except for Digi TV, where the number of TV channels decreased after leaving the business in Croatia. At the end of 2015, the highest number of TV channels were located in the platform Total TV. Since 2010, they were distributed within DTH and first HDTV channels. During 2015 there was a higher growth of HDTV services, so that at the end of 2015 the number of HDTV channels in the platform Total TV were 23 hour Vip 13, Max TV 9, Polaris Media 4. Digi TV to subscribers in Serbia does not offer HDTV services.

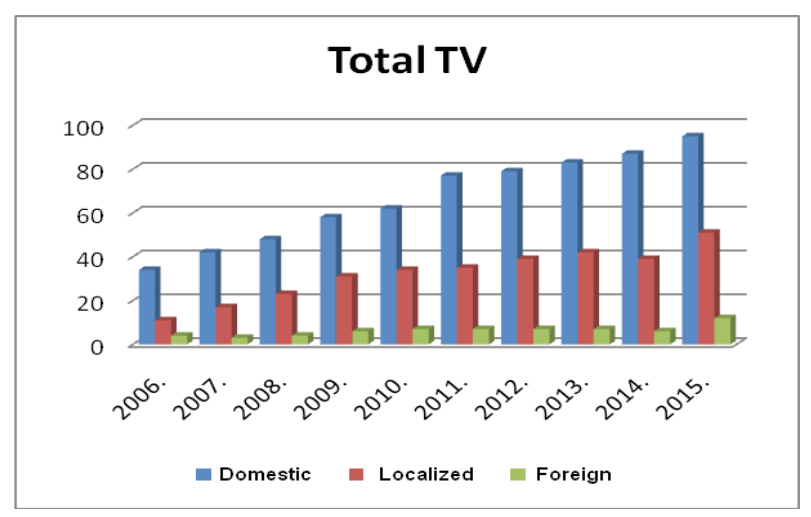

Figure 2. TV channels in DTH platform Total TV.

\begin{tabular}{|c|c|c|c|c|c|c|c|c|c|c|c|}
\hline \multicolumn{2}{|c|}{ DTH provider } & 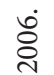 & 옹 & 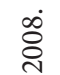 & ஓें & $\stackrel{\dot{0}}{\circ}$ & $\dot{\vec{\Xi}}$ & 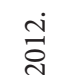 & $\stackrel{\dot{r}}{\vec{D}}$ & 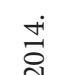 & 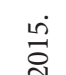 \\
\hline \multirow{2}{*}{ Total TV } & SD & 48 & 62 & 75 & 95 & 100 & 116 & 118 & 125 & 125 & 135 \\
\hline & $\mathrm{HD}$ & - & - & - & - & 3 & 3 & 7 & 7 & 7 & 23 \\
\hline \multirow{2}{*}{ Digi TV } & $\mathrm{SD}$ & 32 & 39 & 44 & 45 & 45 & 43 & 39 & 31 & 29 & 28 \\
\hline & $\mathrm{HD}$ & - & - & - & - & - & - & - & - & - & - \\
\hline \multirow{2}{*}{ Max TV } & SD & - & - & - & - & 50 & 73 & 74 & 72 & 73 & 73 \\
\hline & HD & - & - & - & - & - & 1 & 4 & 5 & 5 & 9 \\
\hline \multirow{2}{*}{ Polaris Media } & $\mathrm{SD}$ & - & - & - & - & 43 & 49 & 58 & 62 & 63 & 63 \\
\hline & $\mathrm{HD}$ & - & - & - & - & 2 & 2 & 2 & 3 & 3 & 4 \\
\hline \multirow{2}{*}{ Vip Sat } & $\mathrm{SD}$ & - & - & - & - & - & - & - & 60 & 61 & 65 \\
\hline & $\mathrm{HD}$ & - & - & - & - & - & - & - & 6 & 6 & 13 \\
\hline
\end{tabular}

Table 4. SDTV and HDTV channels number of DTH providers in countries of Former Yugoslavia 


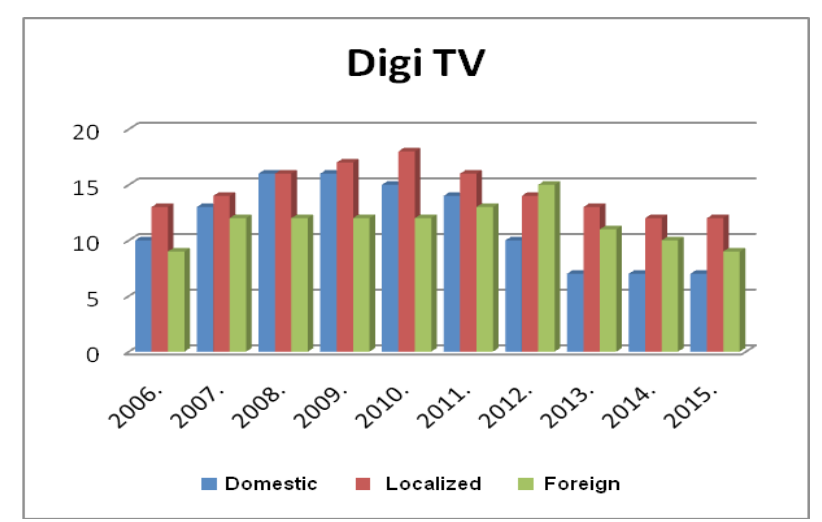

Figure 3. TV channels in DTH platform Digi TV.

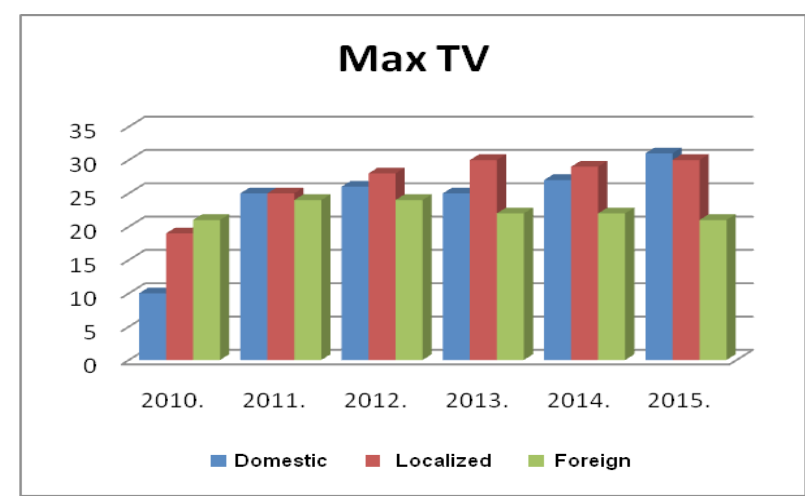

Figure 4. TV channels in DTH platform Max TV.

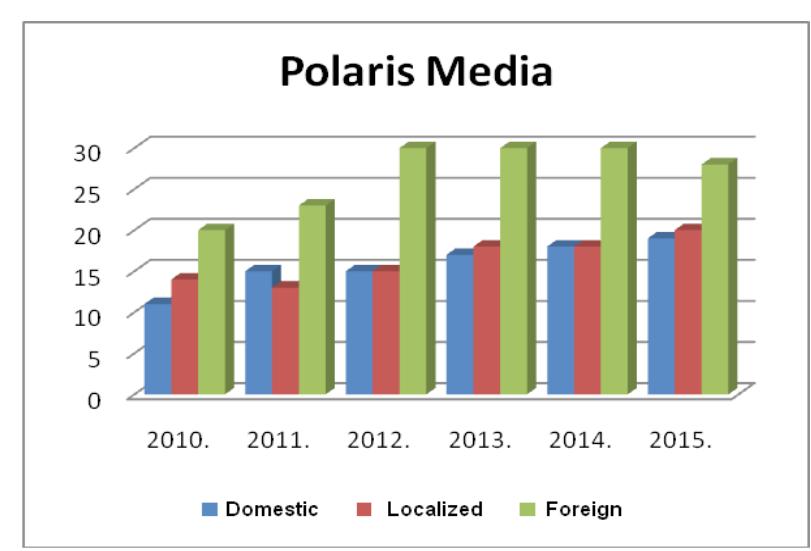

Figure 5. TV channels in DTH platform Polaris Media.

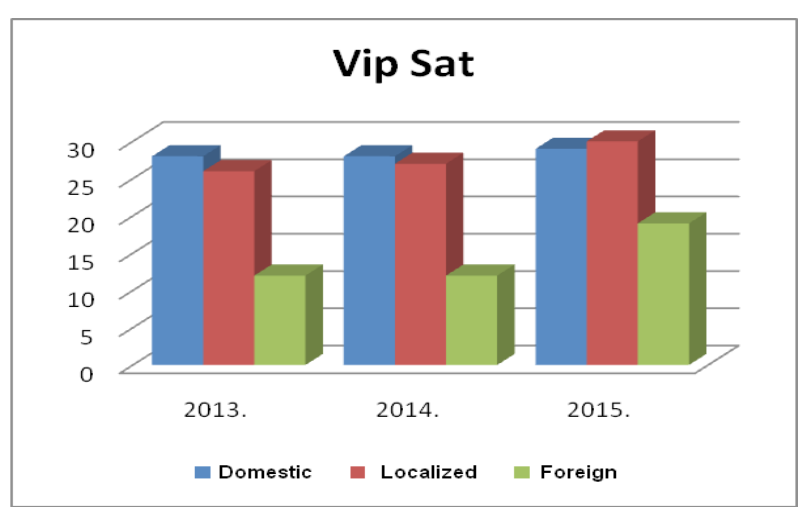

Figure 6. TV channels in DTH platform Vip Sat.
The structure of TV channels that broadcast in regional DTH platform can be classified into three categories: domestic, localized and foreign. Under localized TV channels are considered foreign TV channels (pan-European) that are localized in local languages by DVB subtitles or audio. TV channels that include program broadcast in foreign languages, mainly English, are considered foreign TV channels. The structure of TV channels in DTH platform Total TV, Digi TV, Max TV, Polaris Media and Vip Sat are respectively given in Figure 3, Figure 4, Figure 5 and Figure 6. In Total TV prevails domestic over localized TV channels, and at the minimum are represented foreign TV channels without localization. In Polaris Media, the most represented are foreign TV channels. In other DTH providers, domestic and localized TV channels are equally represented, followed by foreign TV channels.

\section{CONCLUSION}

Direct-to-Home (DTH) refers to the broadcasting of TV programs and related services in which the subscribers, or the final users, receive signals directly from satellite from the geostationary orbit. The signals are broadcasted in digital format and in the Ku band (in Europe). Companies that provide DTH services, provide other services besides TV channels, such as radio, video on demand and satellite internet. DTH services are popular in rural areas where conventional cable services are not available. DTH services are also used by the urban and suburban users, who are not satisfied with the quality and quantity of TV services available by cable operators. In Europe, in the second half of the 1990s, the first DTH services began to operate in digital format, reaching their rapid extension during the 2000s. However, in recent years, rapid expansion of HDTV channels has occurred, which will be replaced by SDTV in the upcoming years. In 2015, the distribution of the first UHD TV channels also occurs, for which the satellite is an ideal way of distribution because of the required large-capacity for transmission (Jakšić et al., 2015).

\section{REFERENCES}

Chaplin, J. (1992). Development of satellite TV distribution and broadcasting. Electronics \& Communication Engineering Journal, 4(1), 33-41.

Digi TV. (2015). URL: www.digi.tv/

Haykin, S. Telecommunication Systems and Technology - Vol.1: Analog and digital transmission of data, URL: http://www.eolss.net/Sample-Chapters/C05/ E6-108-06.pdf. 
Jakšić, B., Petrović, M., Spalević, P., Ivković, R. \& Marković, A. (2014). Deset godina satelitske HDTV u Evropi. Zbornik radova XIII međunarodnog naučno-stručnog simpozijuma INFOTEH 2014, 19-21 mart 2014 (pp. 435-440). Jahorina, Bosna i Hercegovina.

Jaksic, B. Petrovic, M. Milosevic, I., Ivkovic, R. \& Bjelovic, S. (2015). UHDTV into Terrestrial and Satellite Systems. Proceedings of International Scientific Conference UNITECH 2015, 20-21 November 2015 (pp. II112-II118). Gabrovo, Bulgaria.

King Of Sat spisak satelita. (2015). URL: http://en.kingofsat.net/satellites.php.

Ling Sat spisak satelita. (2015). URL: http://www.lyngsat. $\mathrm{com} /$.

Marcotte, S. (2012). The road to UHDTV. Qubeck: Miranda Technolog.

Mirabito, M. \& Morgenstern, N. (2004). Satellites: Operations and Applications, The New Communication Technologies. Burlington: Focal Press.

Mignone, V. Vazquez-Castro, M. A. \& Stockhammer, T. (2011). The Future of Satellite TV: The Wide Range of Applications of the DVB-S2 Standard and Perspectives. Proceedings of the IEEE, 99(11), 1905 1921.

Pattan, B. (1993). Satellite Systems: Principles and Technologies. Berlin: Springer Science \& Business Media.

Pechard, S., Carnec, M., Le Callet, P. \& Barba, D. (2006). From SD to HD television: effects of H.264 distortions versus display size on quality of experience. In IEEE International Conference on Image Processing, 2006 (pp. 409-412). Atlanta, USA.

Petrović, M. (2007). Televizija. Kosovska Mitrovica: FTN.

Petrović, M., Jakšić, B., Spalević, P., Milošević, I. \& Lazić, Lj. (2014). The development of digital satellite television in countries of the former Yugoslavia. Technical Gazette, 21(4), 881-887.

Tirro, S. (1993). Satellite Communication Systems Design. Berlin: Springer Science \& Business Media.

Valenti, M. C. (2011). Modern Digital Satellite Television: How It Works.

VipSat. (2015). URL: www.vipnet.hr/satelitska-televizija. 